Ann. Sci. forest., 1978, 35 (3), 175-185.

\title{
Influence de la photopériode sur l'épaisseur des membranes et le diamètre des lumens des trachéides de quatre essences résineuses
}

\author{
R. KELLER
}

Station de Recherches sur la Qualité des Bois, Centre national de Recherches forestières, INRA, Champenoux, 54280 Seichamps

\begin{abstract}
Résumé
On a étudié l'influence d'une période de jours longs sur l'épaisseur des membranes et le diamètre des lumens des trachéides de quatre essences résineuses : Pseudotsuga menziesii, Picea excelsa, Abies grandis, Abies nordmanniana.

Les mesures ont été faites à l'aide d'un analyseur de texture sur des coupes microscopiques de tiges de plants de 4 ans.

Seul Abies nordmanniana semble n'avoir pas réagi au traitement.
\end{abstract}

\section{1. - Introduction}

La qualité d'un bois est le reflet des conditions qui ont présidé à la croissance de l'arbre qui l'a produit. Les études de physiologie qui, jusqu'ici, ont essentiellement porté sur de jeunes plants, surtout résineux, ont déjà permis de mettre en évidence l'influence de certains facteurs : disponibilité en eau, photopériode, ébourgeonnement, ablation de feuilles ou d'aiguilles sur les caractéristiques des cellules élaborées par l'assise génératrice libéro-ligneuse (P. R. Larson, 1960, 1963, 1964, 1969 ; S. D. Richardson, 1964 ; B. F. Wilson, 1970).

Nous avons pu disposer il y a quelques années de jeunes plants de quatre essences résineuses dont certains avaient subi en 1967 une période de jours longs dans un dispositif de la Station d'Amélioration des Arbres Forestiers.

Des mesures d'épaisseurs de membranes et de diamètres de cellules ont été faites sur des coupes minces provenant de ces plants. L'objet de cette note est de présenter quelques résultats faisant apparaître des différences de dimensions liées à deux types de photopériode : jours normaux d'une part, et jours longs d'autre part. 


\section{2. - Matériel et Méthode}

\section{1. - Description sommaire des échantillons}

Des plants de Pseudotsuga menziesii, Picea excelsa, Abies grandis et Abies nordmanniana avaient été installés dans une bâche à la pépinière d’Amance le 9 mai 1967.

A partir du 17 mai et jusqu'au 13 octobra 1967, une zone de la bâche reçut chaque nuit un supplément d'éclairage d'une heure, entre 0 h et $1 \mathrm{~h}$. Cette interruption de la nuit est équivalente, lorsque les jours sont courts, à un effet photopériodique identique à celui des jours longs (jours longs physiologiques) bien que les plants ne bénéficient pas de $18 \mathrm{~h}$ ininterrompues d'éclairement. On peut donc penser que toute la saison de végétation 1967 a été pour la zone à nuit interrompue une période de croissance en jours longs.

L'autre partie de la bâche, servant de témoin, reçut la lumière normale du jour.

Dans chaque zone, chacune des essences avait été plantée en quatre répétitions.

Les jeunes arbres furent arrachés au début de 1970. Ils étaient alors âgés de 4 ans.

Pour chaque essence, des coupes transversales de $30 \mu \mathrm{m}$ d'épaisseur furent effectuées sur dix plants de chaque répétition dans chaque zone. Ces coupes se situaient au-dessus du collet; elles furent colorées à la safranine.

\section{2. - Méthode de la mesure des membranes et des lumens des trachéides}

Chaque coupe mince, divisée en quadrants, fut analysée automatiquement par l'analyseur de texture Microvideomat (fig.1) que la Société Zeiss nous avait aimablement prêté (R. Keller et $F$. Thiercelin, 1975). Cet appareil nous a fourni le nombre de cellules mesurées dans le champ, la surface des membranes et des lumens.

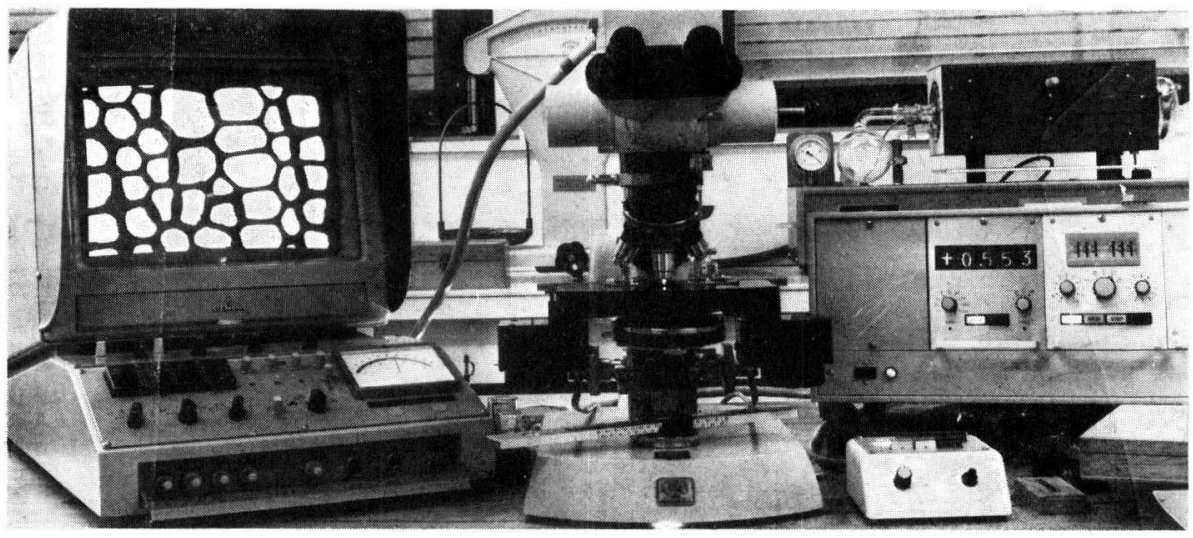

FIG. 1. - Photographie du microvidéomat en fonctionnement.

- Au centre, le microscope, surmonté de la caméra partiellement visible.

- A gauche, l'écran de télévision où apparaissent les lumens (taches claires) et les membranes (zone sombre).

- A droite, le compteur.

Photograph of the working analyser.

- In the middle, the microscope, and, above partially seen, the camera.

- On the left, the television screen on which the lumina (light areas) and the cell walls (dark area) appear.

- On the right, the counter. 
Pour chaque coupe, nous disposons de quatre valeurs du nombre des cellules, de la surface des membranes et de la surface des lumens pour le bois initial, intermédiaire et final des années 1967 et 1968.

Si l'on considère qu'au moins 40 cellules en moyenne ont pu être mesurées dans chaque champ de l'appareil, c'est 1 (zone) $\times 4$ (répétitions) $\times 10$ (arbres) $\times 4$ (quadrants) $\times 3$ (types de bois) $\times 40$ (cellules), soit 19200 cellules qui ont été mesurées pour chaque zone, chaque année et chaque essence. Au total, comme il y a deux zones, deux années et quatre essences, c'est un minimum de 307000 cellules environ qui ont été prises en compte pour l'ensemble de l'expérience.

\section{3. - Mode de détermination du rayon des lumens et de l'épaisseur des membranes}

Le champ rectangulaire de l'appareil correspondait à une surface exploréesur la coupe de largeur $a=95,5 \mu \mathrm{m}$ et de longueur $b=136 \mu \mathrm{m}$, soit une surface réelle de $12988 \mu \mathrm{m}^{2}$.

L'appareil ne permettant pas alors de reconnaître facilement la forme des cellules, les mesures ont été faites en 1972 et depuis les possibilités de ces analyseurs ont été multipliées, on a supposé que tous les éléments mesurés étaient circulaires.

Dans ces conditions, si v est le rapport de la surface totale des lumens à la surface totale explorée, et si $n$ est le nombre total de cellules dans le champ, on peut écrire :

1) surface des lumens plus membranes $=a . b$;

2) surface des membranes $=a \cdot b-v \cdot a \cdot b$;

3) surface des membranes d'une cellule $=\frac{(l-v) \cdot a \cdot b}{n}$;

4) surface du lumen d'une cellule $=\frac{v \cdot a \cdot b}{n}=\pi R_{i}^{2}$ si $R_{i}$ est le rayon du lumen d'où

$$
R_{i}=\sqrt{\frac{v \cdot a \cdot b}{n \cdot \pi}}
$$

5) surface totale d'une cellule $=\frac{a \cdot b}{n}=\pi R_{t}^{2}$ si $R_{t}$ est le rayon de la cellule d'où

$$
R_{t}=\sqrt{\frac{a \cdot b}{n \cdot \pi}} ;
$$

6) épaisseur de la membrane d'une cellule :

$$
e=R_{t}-R_{i}=\sqrt{\frac{a \cdot b}{n \cdot \pi}}(1-\sqrt{v)} .
$$

\section{3. - Résultats}

Ils sont regroupés dans le tableau 1 qui donne, pour chaque case de haut en bas, la valeur en $\mu \mathrm{m}$ de la variable mesurée, son écart-type et son coefficient de variation. 
TABLEAU 1

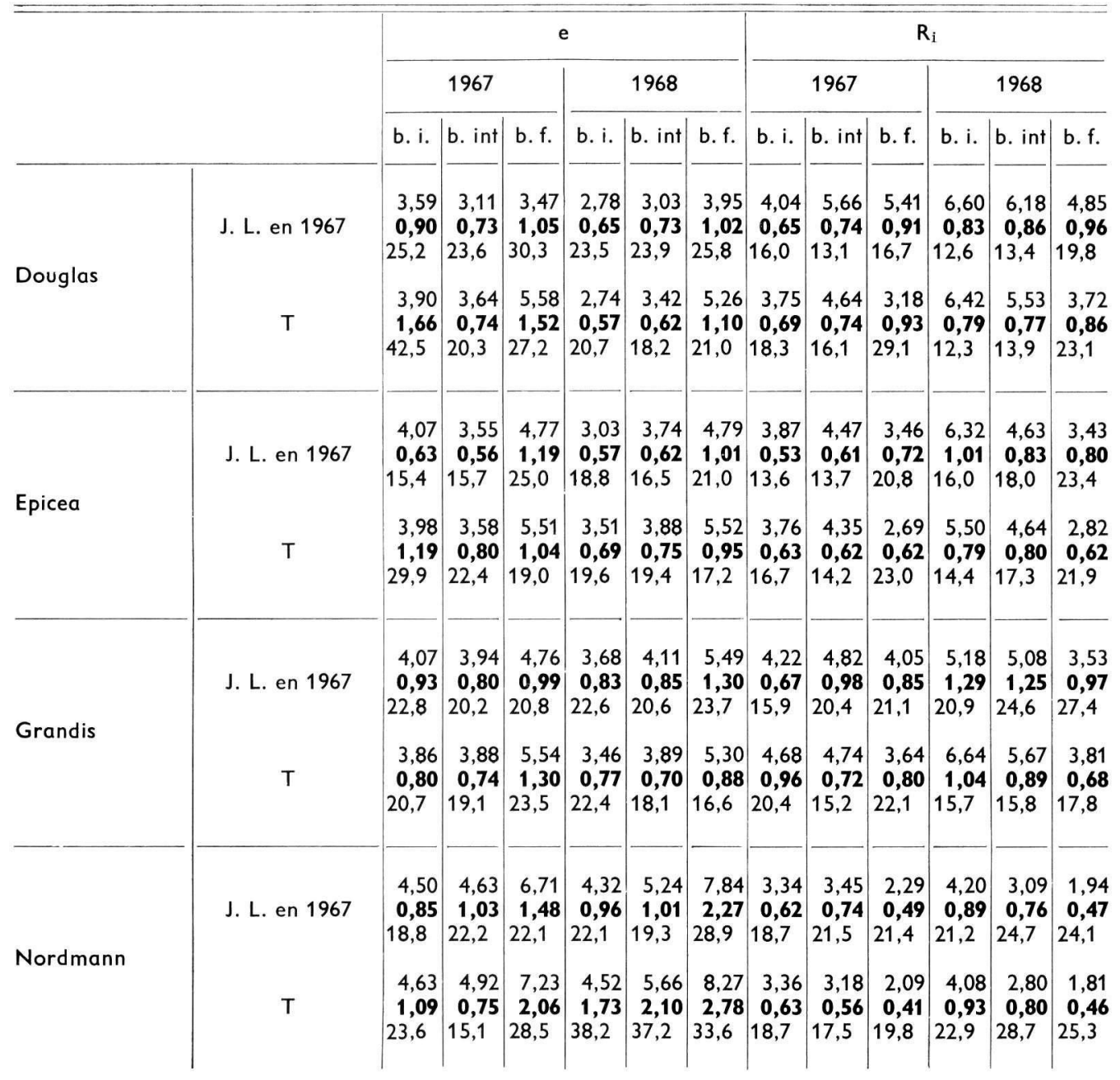

e = épaisseur de la membrane; cell wall thickness.

$\mathbf{R}_{\mathrm{i}}=$ rayon du lumen; radius of the lumen.

b. i. = bois initial ; earlywood.

b. int. = bois intermédiaire ; transitional wood.

b. f. = bois final ; latewood.

J. L. = jours longs en 1967 ; long days treatment in 1967.

$\mathrm{T}=$ témoins; untreated.

Dans chaque case :

1 re ligne: dimension en $u \mathrm{~m}$;

2 e ligne: écart-type en $\mu \mathrm{m}$;

3e ligne : coefficient de variation en p. 100.

In each compartment :

1st line : dimension in $\mu \mathrm{m}$;

2nd line: standard deviation in $\mu \mathrm{m}$;

3rd line : coefficient of variation (percentage). 


\section{1. - Pseudotsuga menziesii}

3.11. Epaisseur de la membrane.

Chez les arbres ayant subi les jours longs (J. L.), l'épaisseur de la membrane n'a pas varié sensiblement en 1967 : entre le bois initial et le bois final, il n'y a pas de différence significative. Les témoins $(T)$ ont suivi l'évolution normale, c'est-à-dire que l'épaisseur des membranes du bois final est très supérieure à celle du bois initial. Les valeurs de l'épaisseur sont significativement différentes entre J. L. et T. pour le bois intermédiaire et le bois final (fig. 2).

En 1968, en l'absence de tout traitement, les arbres ayant subi les jours longs en 1967 et les témoins présentent le schéma normal d'augmentation de l'épaisseur entre le bois initial et le bois final.

\subsection{Rayon des lumens.}

Les rayons $R_{i}$ des lumens des arbres à J. L. sont en 1967 significativement supérieurs à ceux des témoins. Au contraire de ces derniers qui suivent l'évolution normale, c'est-à-dire une diminution entre le bois initial et le bois final, les arbres à J. L. voient ce rayon augmenter entre le début et la fin de la saison de végétation.

En 1968, bien qu'il subsiste pour le bois intermédiaire et le bois final des diffétences significatives entre J. L. et T., les deux modalités suivent l'évolution normale c'est-à-dire que $R_{i}$ diminue.
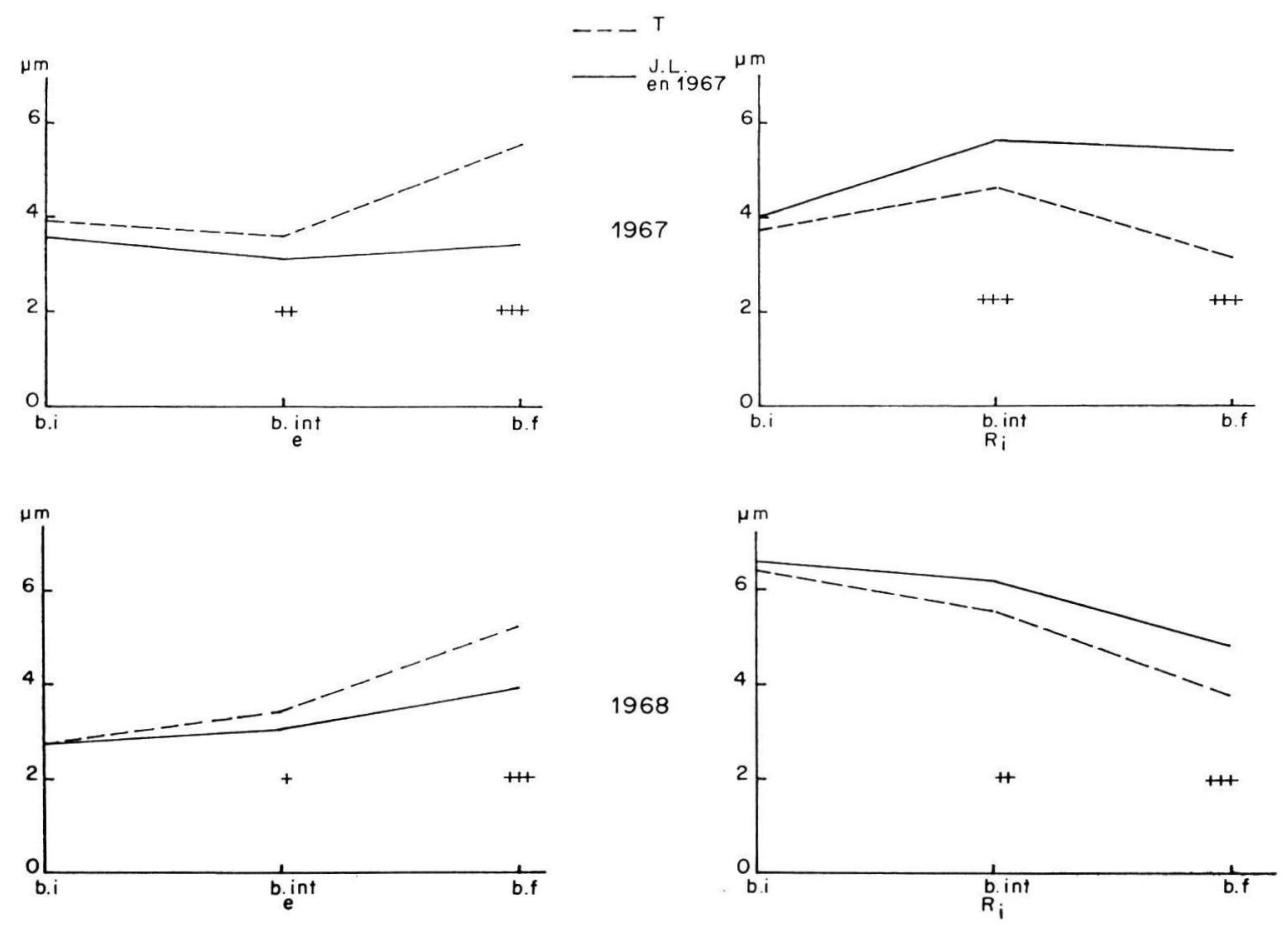

FIG. 2 
R. KELLER
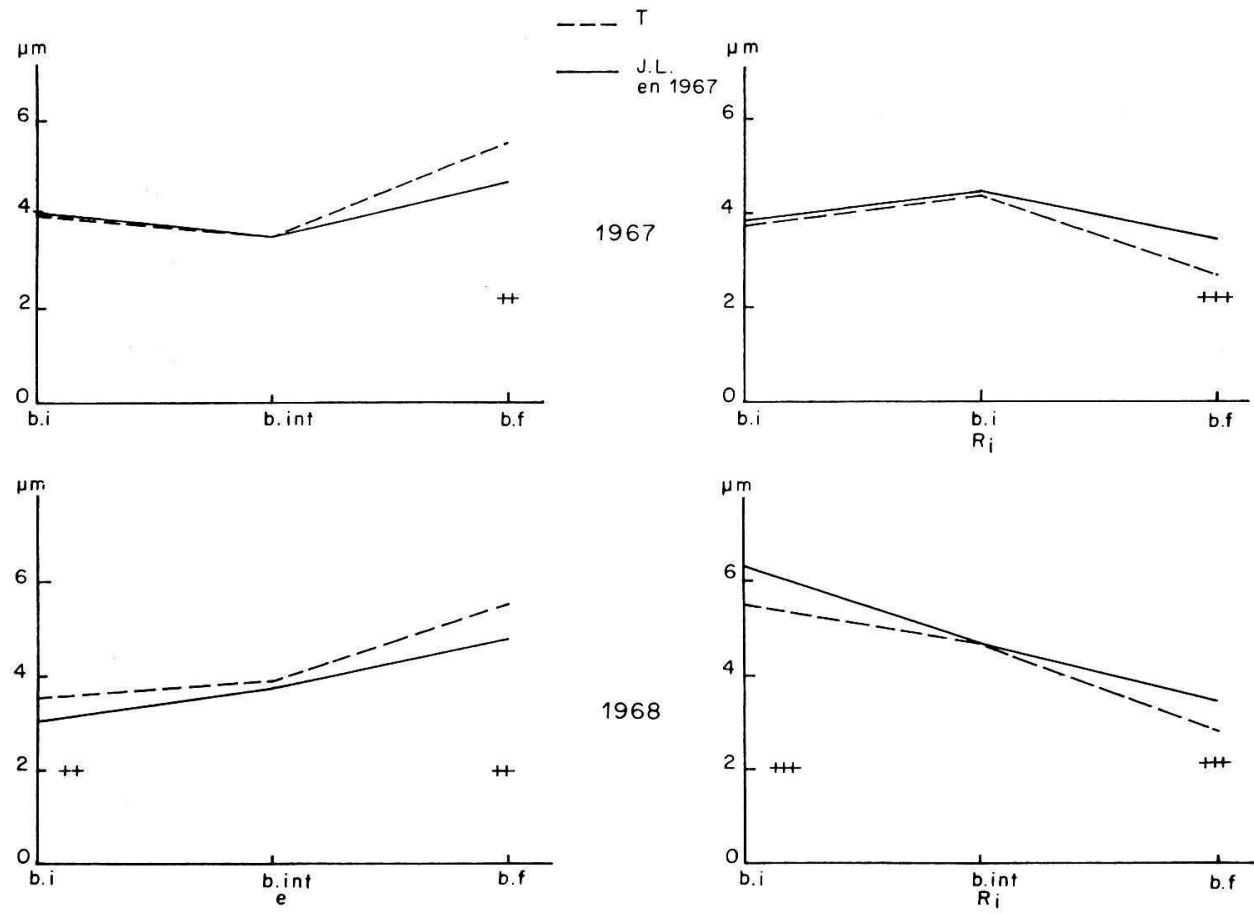

1968

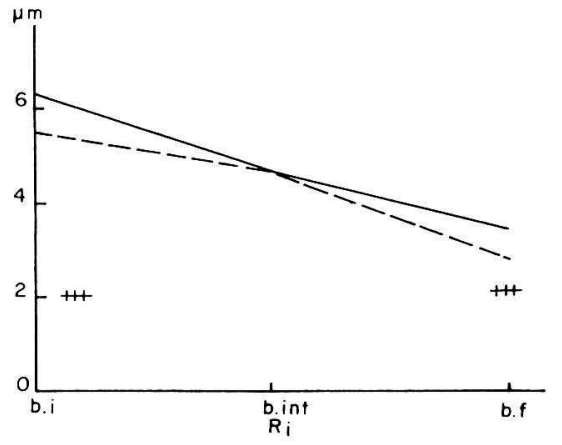

FIG. 3
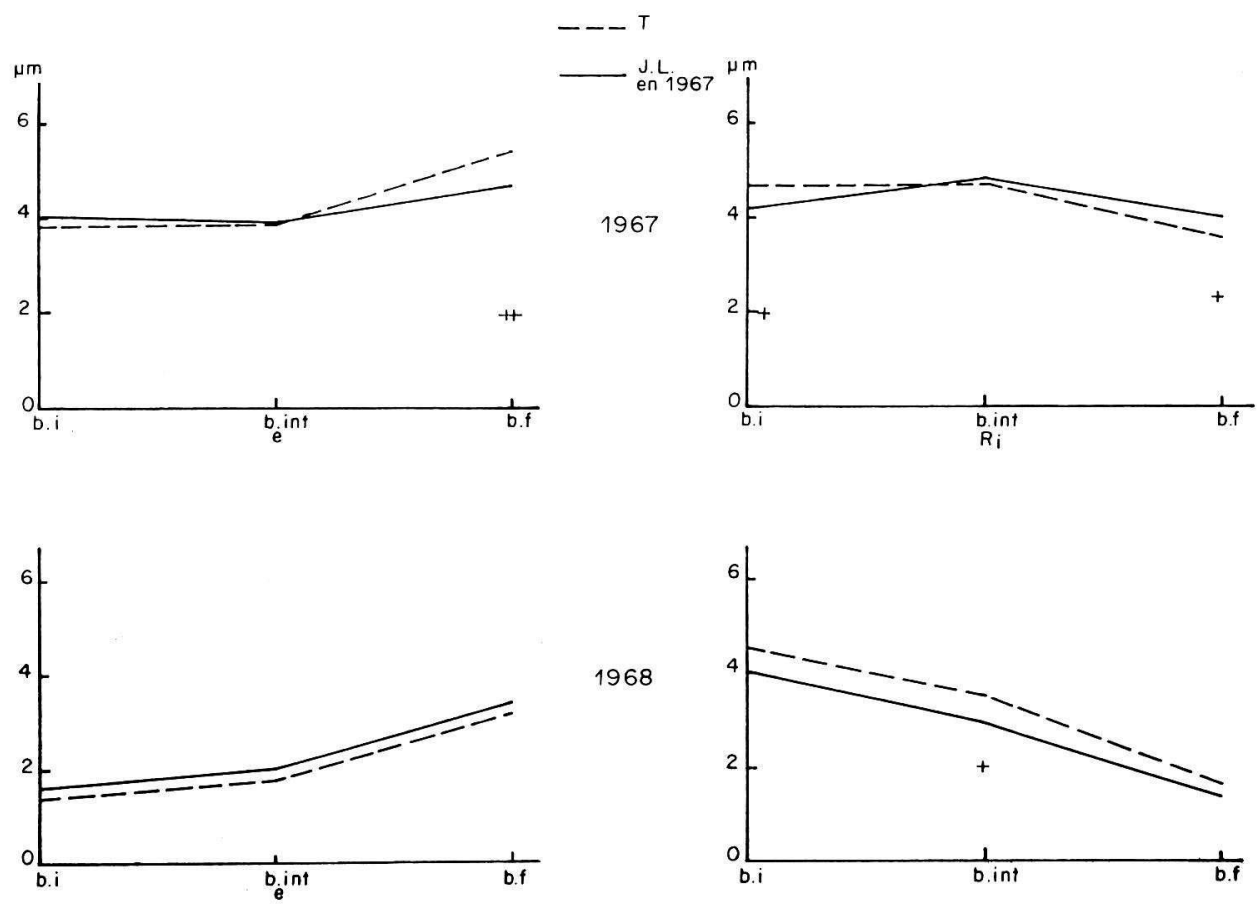

FIG. 4 

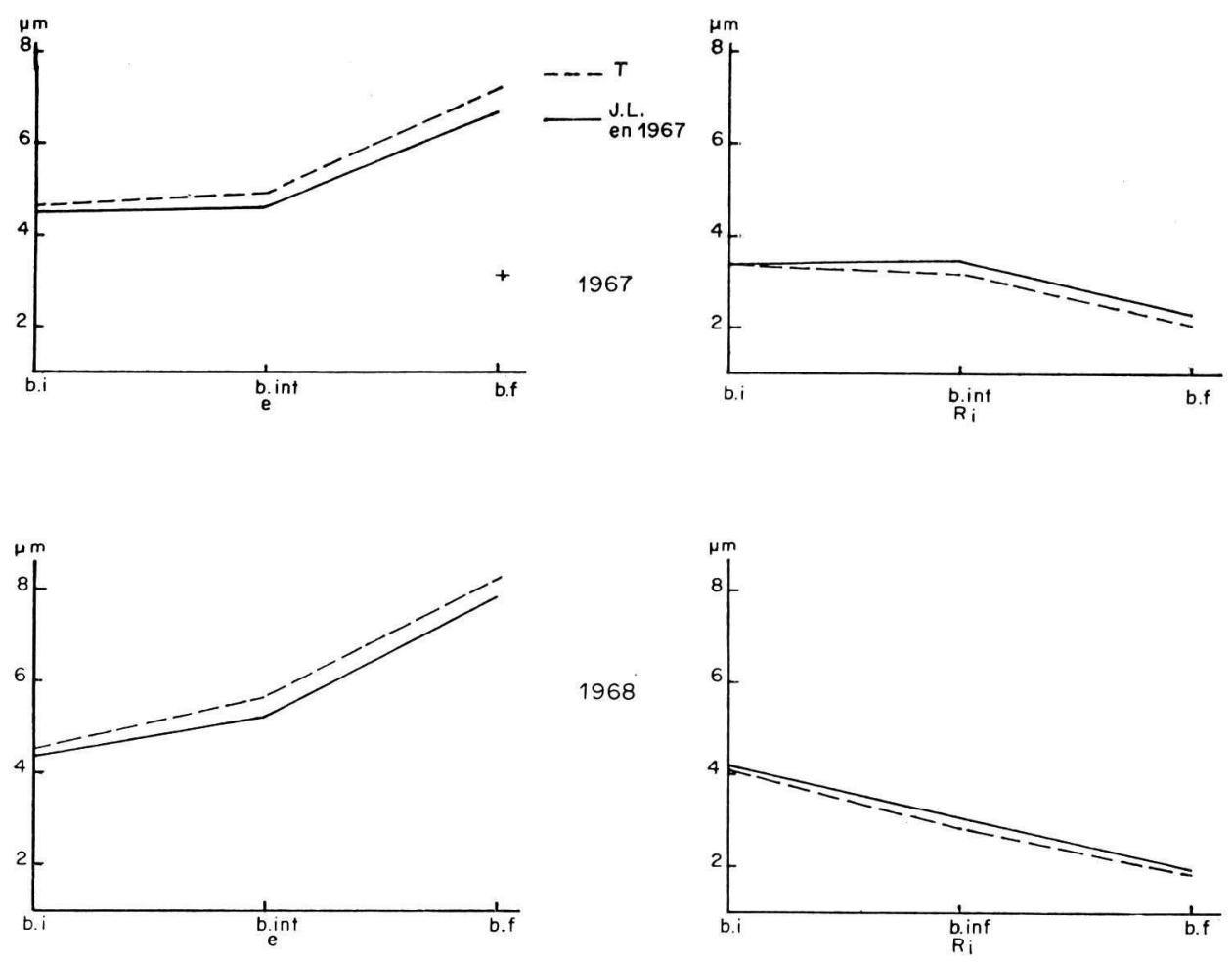

FIG. 5

FIG. 2, 3, 4 et 5. - Graphiques montrant l'évolution de l'épaisseur de la membrane (e) et du rayon du lumen $\left(R_{i}\right)$ en 1967 et 1968.

Graphs showing the evolution of the cell wall (e) and the lumen radius $\left(R_{i}\right)$ during 1967 and 1968.

$\mathrm{T}=$ traitement témoin ; untreated.

J. L. = traitement en jours longs en 1967 ; long days treatment in 1967.

b. i. = bois initial ; earlywood.

b. int. = bois intermédiaire ; transitional wood.

b. f. = bois final ; latewood.

Sevils de signification : * 5 p. 100

** 1 p. 100

*** 1 p. 1000.

* significant difference at 5 p. 100 confidence level

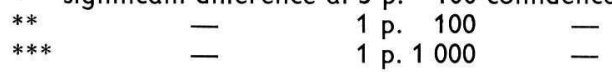

\subsection{Discussion.}

Les arbres à J. L. ont répondu en 1967 au traitement en gardant un fort lumen et une épaisseur de membrane à peu près constante (fig. 6) ; ils n'ont pas exactement suivi le schéma donné par Larson pour les arbres à J. L. qui, entre le bois initial et le bois final, gardent un $R_{i}$ à peu près constant, mais augmentent l'épaisseur de leurs membranes. 
Cette différence de réaction s'explique peut-être par le fait qu'en pépinière, au traitement en photopériode s'est superposée l'influence du milieu extérieur naturel, alors que dans les expériences de Larson, les plants sont placés dans des milieux contrôlés strictement.

II n'en reste pas moins que le traitement en J. L. fait à Amance a marqué nettement les arbres traités et que, lorsqu'il cesse, les cellules retrouvent une évolution classique entre le début et la fin de la saison de végétation.

\section{2. - Picea excelsa}

\subsection{Epaisseur de la membrane.}

Elle augmente légèrement mais significativement du bois initial au bois final chez les arbres à J. L. en 1967 ; cette évolution est moins marquée que chez les témoins (fig. 3), une différence nette apparaissant dans le bois final entre les deuxtraitements.

En 1968, l'épaisseur augmente constamment entre les deux périodes et pour les deux modalités.

\subsection{Rayon du lumen.}

En 1967 et comme pour l'épaisseur, le rayon du lumen des arbres à J. L. diminue nettement entre le bois intermédiaire et le bois final pour aboutir un peu en dessous de sa dimension de départ dans le bois initial. Les témoins ont une évolution semblable, mais beaucoup plus marquée en fin de saison de végétation.

En 1968, le lumen diminue constamment pour les deux traitements entre le bois initial et le bois final.

\subsection{Discussion.}

La réaction de l'épicéa soumis aux J. L. dont l'épaisseur des membranes augmente alors que le lumen ne diminue que faiblement ressemble à celle qui est citée par Larson à propos du type de cellule de jours longs.

La différence avec les témoins se manifeste dans l'aspect du bois final (fig. 7), là où les différences entre les deux traitements sont significatives.

En 1968, des différences subsistent entre les deux modalités, mais elles obéissent toutes deux au schéma classique d'évolution des cellules entre le début et la fin de la saison de végétation.

\section{3. - Abies grandis}

\subsection{Epaisseur de la membrane.}

En 1967, cette essence suit à peu près la même évolution que l'épicéa, les arbres à J. L. aboutissant à une épaisseur moindre que les témoins (fig. 4).

En 1968, l'évolution est normale et la même pour les deux traitements.

\subsection{Rayon du lumen.}

Pour les arbres à J. L., il n'y a pas de différence significative dans les dimensions des lumens entre le début et la fin de la saison de végétation de 1967.

En 1968, l'évolution est parallèle et classique pour les deux modalités. 


\subsection{Discussion.}

Les réactions de ce sapin sont comparables à celles de l'épicéa bien que les différences entre arbres à J. L. et témoins soient moins marquées (fig.8).

\section{4. - Abies nordmanniana}

\subsection{Epaisseur de la membrane.}

En 1967 et 1968, l'évolution des arbres traités et des témoins est classique ; seule une légère différence significative entre eux peut être observée dans le bois final de 1967 (fig. 5).

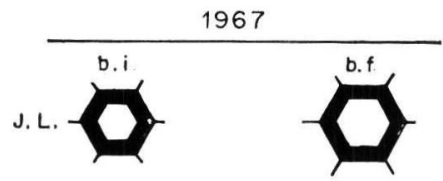
$\overline{10 \mu \mathrm{m}}$ 1968

Fig. 6
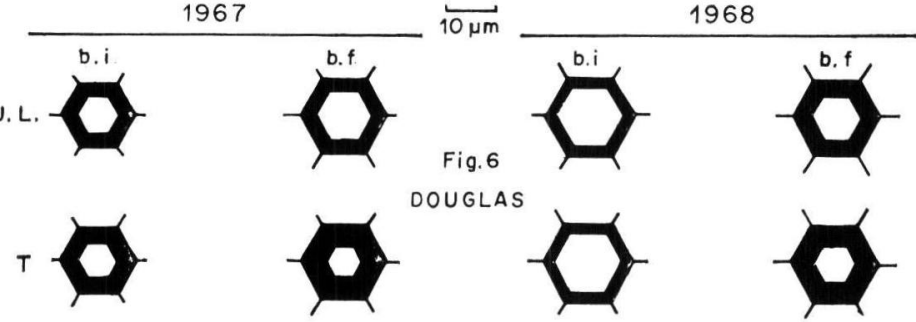

DOUGLAS
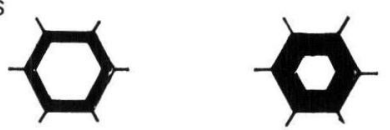

J. L.
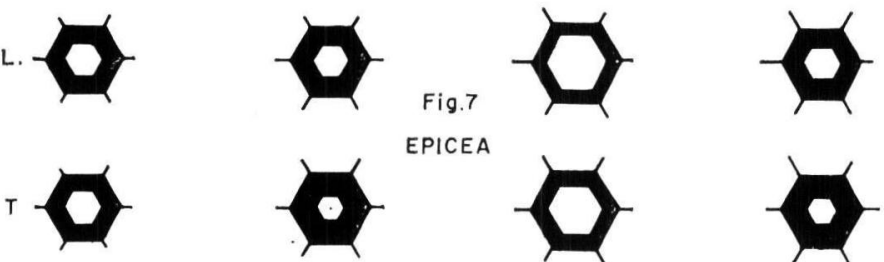

EPICEA
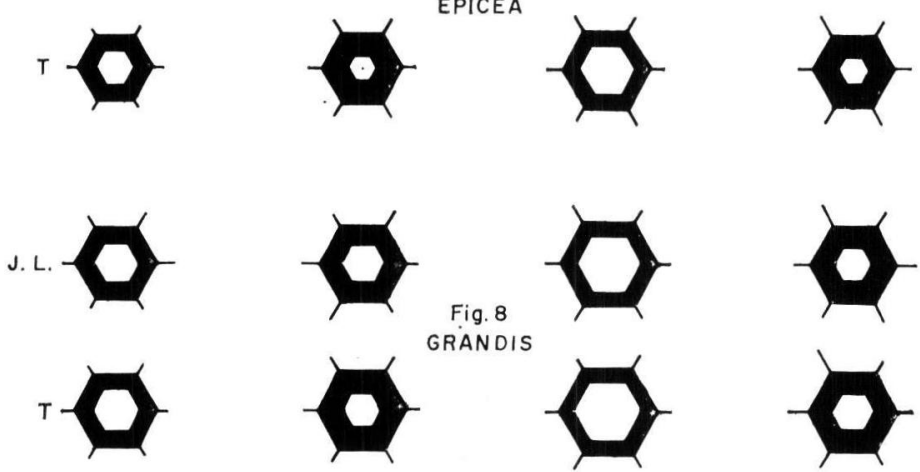

GRANDIS
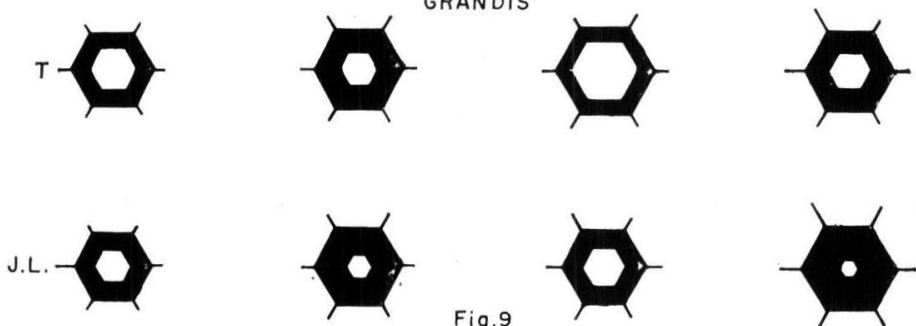

Fig.9
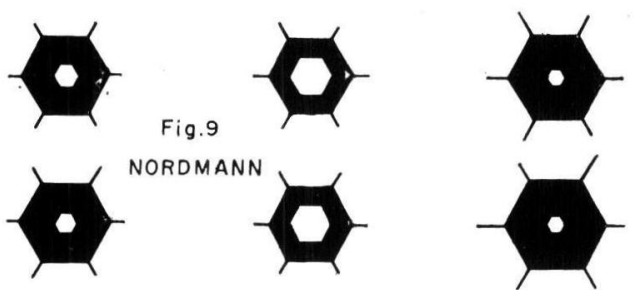

FIG. $6,7,8$ et 9 . Représentation schématique des cellules Schematic patterns of cells. 
3.42. Rayon du lumen.

Au cours des deux années, son évolution est normale et la même pour les deux traitements.

\subsection{Discussion.}

II ne semble pas que le régime de photopériode adopté en 1967 ait eu une quelconque influence sur le sapin de Nordmann (fig. 9).

\section{4. - Conclusion}

Cette expérience montre que sur le plan anatomique, les jours longs ont marqué trois des quatre essences résineuses.

Le douglas semble avoir été le plus sensible, sans toutefois présenter le type de bois final de jours longs cité par Larson.

L'épicéa et le sapin géant ont été moins sensibles, mais les cellules de bois final de jours longs seraient plus conformes au type correspondant de Larson.

Le sapin de Nordmann semble n'avoir pas réagi à la photopériode telle qu'elle a été définie dans cette expérience.

Il est possible que les différences entre les types de cellules observés et ceux cités dans la littérature proviennent de modalités différentes de photopériode et du fait, qu'en pépinière, on ne contrôle pas les autres facteurs comme on peut le faire en serres ou en chambres climatisées.

Il est enfin inutile d'insister sur l'intérêt de l'emploi d'un analyseur de texture dans de telles études anatomiques consécutives à des expériences sur la physiologie des arbres.

II ne semble pas que les simplifications adoptées relatives à la forme des cellules aient apporté de biais importants. Elles seraient d'ailleurs inutiles dans l'hypothèse de l'utilisation d'appareils actuels qui ont bénéficié depuis l'époque de nos mesures de nombreux perfectionnements.

Reçu pour publication en décembre 1977.

\section{Summary}

Influence of a long day period on the cell wall thickness and the lumen diameter of tracheids of four conifers

The influence of a long day period on the cell wall thickness and the lumen diameter of tracheids of four conifers : Pseudotsuga menziesii, Picea excelza, Abies grandis, Abies nordmanniana has been investigated.

The measurements has been done with a texture analyser working on microscopic transverse sections of the stem of four years old trees.

Abies nordmanniana alone has apparently not reacted to this treatment.

\section{Zusammenfassung}

Wirkung des photoperiodismus auf die dicke der zellwande und den durchschnitt der tracheidenlumina bei vier arten von nadelbaumen

Untersucht wurde die Wirkung einer Zeitspanne langer Tage auf die Dicke der Zellwände und den Durchschnitt der Tracheidenlumina und zwar bei vier verschiedenen Nadelbäumen : Pseudotsuga menziesii, Picea excelsa, Abies grandis, Abies nordmanniana. 
Die Messungen wurden mithilfe eines Texturanalysators an mikroscopischen Dünnschnitten von vierjährigen Stämmchen unternommen.

Abies nordmanniana allein hat anscheinend nicht reagiert.

\section{Références bibliographiques}

KELLER R., HERCELIN F., 1975. Influence des gros rayons ligneux sur quelques propriétés du bois de hêtre. Ann. Sci. forest., 32, 2, 113-129.

LARSON P. R., 1960. A physiological consideration of the springwood summerwood transition in Red Pine. Forest Science, vol. 6, $n^{\circ} 2$, juin.

LARSON P. R., 1963. The indirect effect of drought on tracheid diameter in Red Pine. Forest Science, vol. 9, $n^{\circ} 1$, mars.

LARSON P. R., 1964. Some indirect effects of environment on wood formation. De Formation of wood in Forest Trees. Academic Press Inc., New York.

LARSON P. R., 1969. Wood formation and the concept of wood quality. New Haven, Yale University, School of Forestry, Bulletin $n^{\circ} 74$.

RICHARDSON S. D., 1964. The formation of wood in forest-trees. Forestry, vol. XXXVII, n⿳0 1.

RICHARDSON S. D., 1964. The external environment and tracheid size in conifers. De Formation of Wood in Forest Trees. Academic Press Inc., New York, 1964.

WILSON B. F., 1970. The growing tree. The University of Massachusetts Press, Amherst. 